Research Paper

\title{
A novel IncRNA, RPL34-AS1, promotes proliferation and angiogenesis in glioma by regulating VEGFA
}

\author{
Dongzhi Zhang1,2,3,5, Haiping Jiang1,2,3, Junyi Ye ${ }^{1,2,3}$, Ming Gao ${ }^{1,2,3}$, Xinzhuang Wang1,2,3, Enzhou Lu ${ }^{1,2,3}$, \\ He Yang1,2,3, Lixiang Wang ${ }^{1,2,3}$, Shiguang Zhao ${ }^{1,2,3,4 \bowtie}$ \\ 1. Department of Neurosurgery, The First Affiliated Hospital of Harbin Medical University, Harbin, China. \\ 2. Key Colleges and Universities Laboratory of Neurosurgery in Heilongjiang Province, Harbin, China. \\ 3. Institute of Neuroscience, Sino-Russian Medical Research Center, Harbin Medical University, Harbin, China. \\ 4. Shenzhen University General Hospital, Xueyuan AVE 1098, Nanshan District, 11, Shenzhen, Guangdong, P. R. China. \\ 5. Department of Neurosurgery, The Affiliated Cancer Hospital of Harbin Medical University, Harbin, China. \\ $\bowtie$ Corresponding author: Shiguang Zhao, E-mail: guangsz@hotmail.com \\ (c) The author(s). This is an open access article distributed under the terms of the Creative Commons Attribution License (https://creativecommons.org/licenses/by/4.0/). \\ See http:/ /ivyspring.com/terms for full terms and conditions.
}

Received: 2021.02.12; Accepted: 2021.07.09; Published: 2021.08.27

\begin{abstract}
Purpose: Brain gliomas are the most common primary malignant tumors of the central nervous system and one of the leading causes of death in patients with intracranial tumors. The IncRNA RPL34-ASI is significantly upregulated in glioma tissues. However, the biological function of RPL34-AS1, especially in proliferation in glioma, remains unclear.

Methods: The role of RPL34-AS1 in proliferation and angiogenesis in glioma cells was investigated using the LN229, U87, and U251 glioma cell lines. The levels of RPL34-AS1 were detected using real-time quantitative reverse transcription polymerase chain reaction. CCK-8 and colony formation assays were performed to determine the role of RPL34-AS1 in proliferation and survival, and its role in angiogenesis was assessed by an endothelial tube formation assay. Changes in protein levels were assessed by western blotting.

Results: RPL34-AS1 was upregulated in glioma tissues and was correlated with tumor grade. RPL34-AS1 expression was also higher in glioma cells than in normal astrocytes. Knockdown of RPL34-AS1 blocked glioma cell proliferation by inhibiting angiogenesis. This effect occurred through decreased ERK/AKT signaling.

Conclusions: This study suggests that RPL34-AS1 affects cell proliferation and angiogenesis in glioma and therefore may potentially serve as a valuable diagnostic and prognostic biomarker and therapeutic target in patients with glioma.
\end{abstract}

Key words: lncRNA, RPL34-AS1, glioma, proliferation, angiogenesis, VEGFA

\section{Introduction}

Glioma is one of the most aggressive and common primary tumors of the central nervous system (CNS), accounting for almost $28 \%$ of all primary brain tumors and $80 \%$ of all malignant brain tumors [1]. Glioma is divided into malignancy grades (WHO grade I to IV), which assists with determining the optimal treatment. Grades I and II indicate low-grade glioma (LGG), whereas grades III and IV are high-grade glioma (HGG) and have a worse prognosis [2]. The prognosis for patients who are diagnosed with glioblastoma (WHO grade IV) is bleak, with a median survival time of less than 2 years [3]. Surgery, chemotherapy, and radiotherapy remain the principal treatment modalities for glioma; however, only a small number of patients can achieve good results [4]. Therefore, identifying the pathogenesis of glioma is of great significance for treatment.

Angiogenesis, or the growth of new blood vessels, is a key event that has been shown to be important in tumorigenesis and tumor progression [4]. Angiogenesis plays crucial homeostatic roles, since blood vessels carry nutrients to tissues and organs and remove catabolic products. However, pathological angiogenesis can promote or facilitate numerous disease processes [5]. As a high blood flow-dependent malignant tumor, glioma needs more support for tumor angiogenesis than other tumors [6]. 
Gliomas stimulate the formation of new blood vessels through processes driven primarily by vascular endothelial growth factor A (VEGFA), which is the most important factor for promoting angiogenesis in tumors, including glioma $[7,8]$. Studies have shown that VEGFA is upregulated in glioma [9] and is essential for the development of the disease [10]. Therefore, an increasing number of studies have targeted tumor angiogenesis in the treatment of glioma.

LncRNAs are a type of noncoding RNA with a length $>200$ nucleotides with limited coding potential [11]. LncRNAs are categorized according to their genomic location with respect to nearby protein-coding genes, namely, intergenic lncRNAs, intronic lncRNAs, and antisense lncRNAs [12]. LncRNAs can regulate malignant behaviors in glioma, such as cell proliferation, metastasis, invasion, and drug resistance $[13,14,15]$. Therefore, some lncRNAs have been considered as entry points for glioma treatment. For example, H19 is significantly overexpressed in $\mathrm{CD}_{133^{+}}$glioblastoma cells, which increases neurosphere formation in glioblastoma cells [16]. HOTAIR is another important lncRNA overexpressed in glioma. HOTAIR can promote the malignant behavior of glioma through PI3K/AKT and MEK1/2 signaling by downregulating miR-326 [16]. Ribosomal protein L34 antisense RNA 1 (RPL34-AS1) is a novel lncRNA that inhibits invasion in papillary thyroid cancer cell lines [17] and inhibits esophageal cancer cell proliferation by downregulating RPL34 expression [18]. The same effect has been observed in colorectal and gastric cancers [19,20]. We found increased expression of RPL34-AS1 in glioma; however, its biological effect on glioma remains unknown.

\section{Materials and Methods}

\section{Human glioma specimens}

Human glioma specimens were obtained from patients undergoing initial surgery who were diagnosed with glioma at the Department of Neurosurgery, The First Affiliated Hospital of Harbin Medical University and Department of Neurosurgery, The Affiliated Cancer Hospital of Harbin Medical University between September 2019 and December 2020. The grading of glioma specimens was determined by a neuropathologist according to the 2007 WHO classification. All patients signed an informed consent. The samples were stored at $-80^{\circ} \mathrm{C}$ before being embedded in paraffin. Normal brain tissues were obtained from patients with severe traumatic brain injury who underwent decompression surgery and were used as negative control. The study was approved by the Ethics Committee of The First Affiliated Hospital of Harbin Medical University.

\section{Cell lines and culture conditions}

Human glioma cell lines (A172, U87, U251, LN229 and T98G), normal human astrocytes (NHA), and human umbilical vein endothelia cells (HUVECs) were obtained from the China Infrastructure of Cell Line Resource (National Science \& Technology Infrastructure, NSTI). All cell lines were recently authenticated by STR analysis and tested for mycoplasma contamination. Glioma cell lines and NHA were cultured with Dulbecco's Modified Eagle's Medium (DMEM) containing 10\% fetal bovine serum (FBS). HUVECs were cultured in High-glucose Dulbecco's modified Eagle's medium (DMEM) and DMEM-F12 mixed medium containing 10\% FBS. Routine culture was performed in a humidified incubator maintained at $37{ }^{\circ} \mathrm{C}$ with $5 \% \mathrm{CO}_{2}$ and $95 \%$ air.

\section{Cell transfection}

The Lipofectamine 2000 reagent was used to transfect the glioma cell lines with siRNAs according to the manufacturer's instructions. The siRNAs were purchased from GenePharma (Shanghai, China).

\section{Quantitative real-time PCR (qRT-PCR)}

Total RNA was extracted using TRIzol reagent (Invitrogen, USA). The RNA concentration and quality were determined at $260 / 280 \mathrm{~nm}$ ratio using a NanoDrop spectrophotometer (Thermo Scientific ${ }^{\mathrm{TM}}$ NanoDrop 2000c). Reverse transcription was performed using a PrimeScript RT reagent kit (ToYoBo). A SYBR Green PCR Master Mix kit (Roche, Germany) was utilized to verify the expression of the target gene. qRT-PCR was performed using an ABI 7500 Real-Time PCR. Relative expression was normalized to that of endogenous controls using the comparative cycle threshold method, and the fold change in gene expression was calculated using the


GCTCAC, RPL34-AS1-r: TGATGGCTTCTTCAACCA GGA.

\section{Cell viability assay}

U87, U251, and LN229 cells were treated with siRNAs and then seeded (4000 cells/well) in 96-well plates the next day. Cell proliferation was measured through the CCK-8 assay. After the addition of $10 \mu \mathrm{L}$ of CCK-8 solution, the cells were incubated at $37{ }^{\circ} \mathrm{C}$ for one hour. Absorbance at $450 \mathrm{~nm}$ was measured with a spectrophotometer. The experiment was repeated at least three times. 


\section{Colony formation assay}

U87, U251, and LN229 cells (1000 cells/well) were seeded into 6-well plates and cultured for 10 days. The colonies were fixated in $4 \%$ formaldehyde for 30 minutes and then stained with $0.1 \%$ crystal violet for ten minutes. Viable colonies that contained more than 50 cells were counted. The experiment was performed at least thrice for each cell line.

\section{Flow cytometric analysis of cell apoptosis}

The FITC Annexin V Apoptosis Detection Kit was used to stain the glioma cell lines according to the manufacturer's recommendations. Cell apoptosis was then measured by a FACScan flow cytometry.

\section{Western blot analysis}

Protein samples were extracted from the glioma cell lines by RIPA buffer, which contains phenylmethylsulfonyl fluoride (PMSF) and a phosphatase inhibitor. Protein samples were separated by 7-12.5\% SDS-PAGE gels and transferred to polyvinylidene difluoride (PVDF) membranes. The membranes were blocked with 5\% skim milk and incubated with the primary antibody against VEGFA, actin, Akt, p-Akt, Erk, p-Erk, BCL-2, and BAX at $4{ }^{\circ} \mathrm{C}$ overnight. The membranes were incubated with fluorescent-dye conjugated secondary antibodies at room temperature for two hours the next day. The membranes were observed using a ChemiDoc XRS + Imaging System.

\section{Tube formation assay}

A standard Matrigel assay was performed to evaluate in vitro angiogenesis activity by quantifying the tube formation. The 96-well culture plates were coated with $80 \mu \mathrm{l}$ of Matrigel per well, and then allowed to polymerize for thirty minutes at $37^{\circ} \mathrm{C}$. HUVECs $\left(1.5 \times 10^{4}\right.$ cells/well) were grown in the absence or presence of $100 \%$ conditional medium that the supernatant of the cells was collected and centrifuged at $2,000 \times \mathrm{g}$ for 20 minutes at $4{ }^{\circ} \mathrm{C}$ at $48 \mathrm{~h}$ after the transfection for $6 \mathrm{~h}$ at $37^{\circ} \mathrm{C}$ in a 96-well plate. Each well was photographed at $\times 100$ magnification using microscopy. The total tubule length and number of tubule branches were measured through Image J software.

\section{Statistical analysis}

Statistical analyses comparing data between groups were performed using Student's t-test or one-way ANOVA. The association between target gene and clinical features was analyzed using Pearson's chi-square test. Data are presented as mean \pm standard error of the mean (SEM). $\mathrm{P}<0.05\left(^{*}\right)$ was considered to indicate a statistically significant result.
All statistical analyses were carried out using (Prism software version 7.0).

\section{Results}

\section{RPL34-AS1 is upregulated in glioma tissues and cell lines, and its expression is correlated with tumor grade}

To better understand its role in glioma, we performed analyses from GENECARDS and RNAcentral that RPL34-AS1 is localized to human chromosome 4q25 (Figure 1A) and highly conserved in humans (Figure 1B). The expression of RPL34-AS1 was higher in 518 glioma tissues than in 207 normal tissues (Figure 1C), according to GEPIA data from TCGA and GTEx. We then performed qRT-PCR to verify the expression of RPL34-AS1 in glioma tissues and normal brain tissues. We found high RPL34-AS1 expression in glioma tissue specimens, especially in HGGs, compared to that in normal brain tissue specimens (Figure 1D).

We divided the 56 patients with glioma into a high RPL34-AS1 expression group $(n=34)$ and a low expression group $(n=22)$ (Table 1$)$ and analyzed the correlation between RPL34-AS1 expression and clinicopathological features. As shown in Table 1, high RPL34-AS1 was significantly correlated with tumor grade $(P=0.029)$ but had no significant correlation with age, sex, or KPS (P > 0.05).

Moreover, the expression of RPL34-AS1 in glioma cell lines and normal human astrocytes (NHAs) was also evaluated. We observed notably high expression of RPL34-AS1 in LN229, U87, and U251 cells compared with the expression in NHAs (Figure 1E). Because the expression of RPL34-AS1 was highest in these glioma cell lines, we used LN229, U87, and U251 cells for further experiments.

\section{Knockdown of RPL34-AS1 suppresses proliferation in GBM cell lines}

To explore the functional relevance of RPL34AS1 in glioma cells, we interfered with its expression by transfecting cells with RPL34-AS1 siRNAs. We validated the knockdown of RPL34-AS1 by qRT-PCR (Figure 2A). CCK-8 assays showed that knockdown of RPL34-AS1 impaired the proliferation of glioma cells (Figure 2B). We performed a colony formation assay to assess the effect of RPL34-AS1 on glioma cell survival (Figure 2C). We also tested the expression of the apoptosis-related factors Bcl-2 and Bax by western blot after knockdown of RPL34-AS1 (Figure 2D). Flow cytometry assays also revealed that silencing RPL34-AS1 induced apoptosis in LN229 and U251 glioma cells (Figure 2E). These data suggest an oncogenic role of RPL34-AS1 in glioma progression. 
A

Chr 4

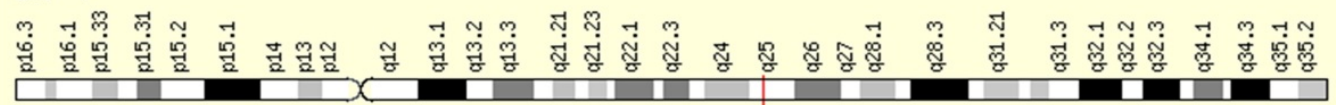

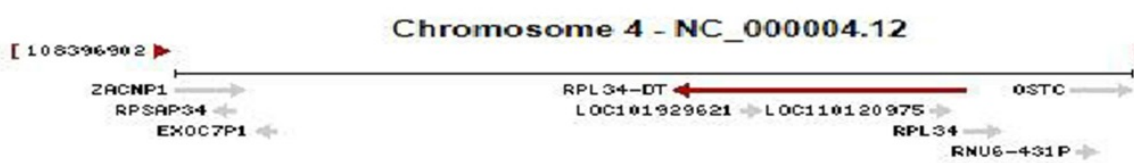

$[108667820$

B

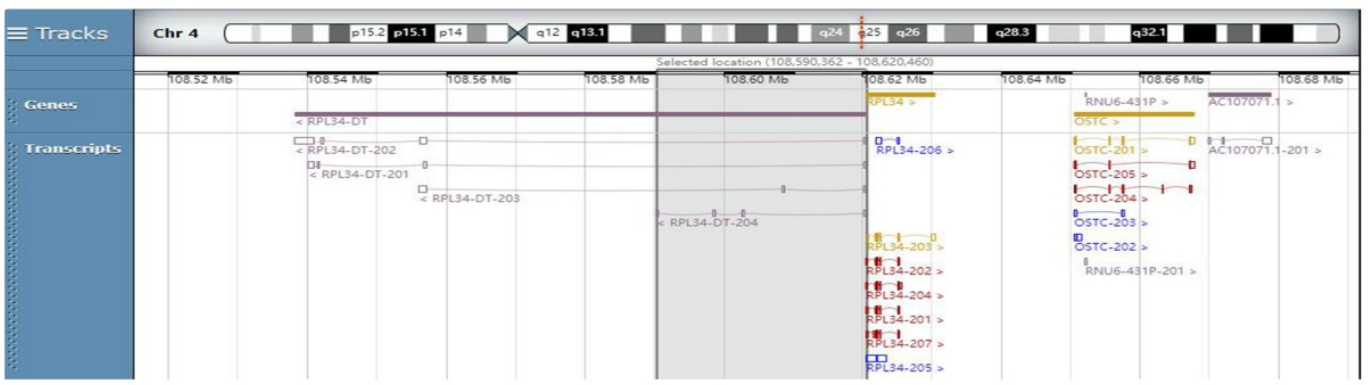

C


=

D

E
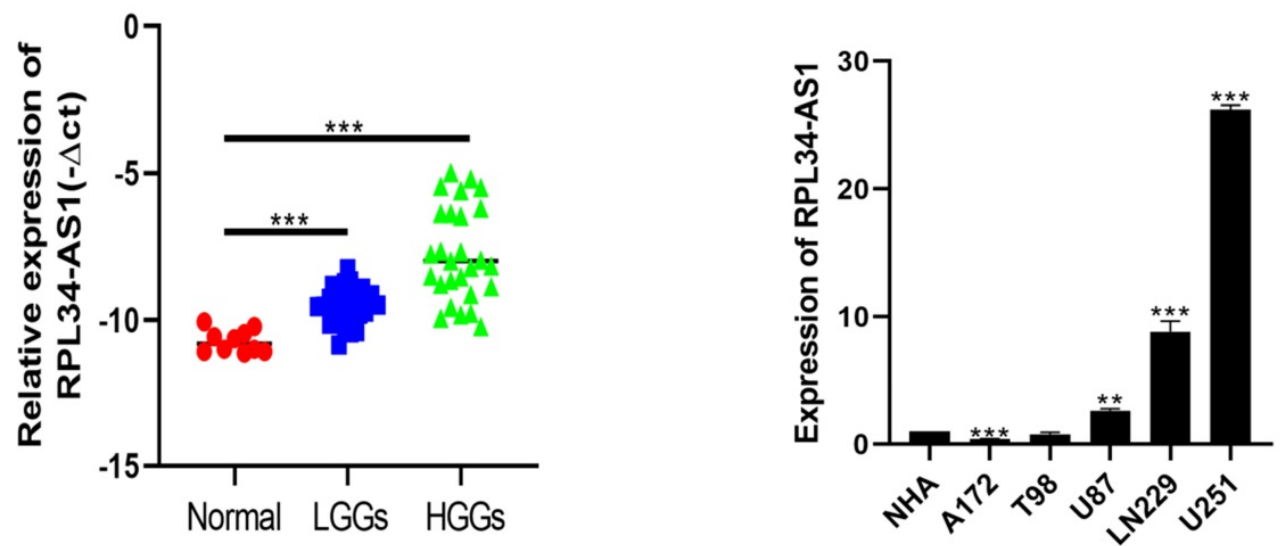

Figure 1. RPL34-AS1 is upregulated in glioma. (A, B) RPL34-AS1 is localized th the human chromosome 4q25 and highly conserved in human. (C) The gene expression profile across all tumor samples and paired normal tissues. RPL34-AS1 is upregulated in glioma tissues compared with normal brain tissues from TCGA and GTEx. (D) The differential expression of RPL34-AS1 in glioma specimens of different grades and normal brain tissue specimens. (E) The expression of RPL34-AS1 examined by qPCR in five glioma cell lines and normal human astrocytes cell line (NHA). $* \mathrm{P}<0.05 ; * * \mathrm{P}<0.01 ; * * * \mathrm{P}<0.001$. 
Table 1. Correlation between IncRNA RPL34-ASI expression level and clinicopathological characteristics in glioma patients ( $\mathrm{n}=56$ )

\begin{tabular}{|c|c|c|c|c|c|}
\hline \multirow[t]{2}{*}{ Features } & \multirow{2}{*}{$\begin{array}{l}\text { No. of } \\
\text { patients }\end{array}$} & \multicolumn{2}{|c|}{ RPL34-AS1 expression } & \multirow[t]{2}{*}{ Ratio (High/All) } & \multirow[t]{2}{*}{ P-value } \\
\hline & & & & & \\
\hline \multicolumn{6}{|l|}{ Age } \\
\hline$<45$ & 39 & 20 & 19 & 0.513 & \multirow{2}{*}{0.217} \\
\hline$\geq 45$ & 17 & 6 & 11 & 0.353 & \\
\hline \multicolumn{6}{|l|}{ Gender } \\
\hline Male & 31 & 17 & 14 & 0.548 & \multirow{2}{*}{0.678} \\
\hline Female & 25 & 18 & 7 & 0.720 & \\
\hline \multicolumn{6}{|l|}{ KPS } \\
\hline$\geq 80$ & 30 & 19 & 11 & 0.633 & \multirow{2}{*}{0.523} \\
\hline $\begin{array}{l}<80 \\
\text { Grade }\end{array}$ & 26 & 12 & 14 & 0.462 & \\
\hline $\operatorname{Low}(I+I I)$ & 29 & 14 & 15 & 0.483 & \multirow[t]{2}{*}{$0.029^{*}$} \\
\hline High $(I I I+I V)$ & l) 27 & 20 & 7 & 0.741 & \\
\hline
\end{tabular}

A
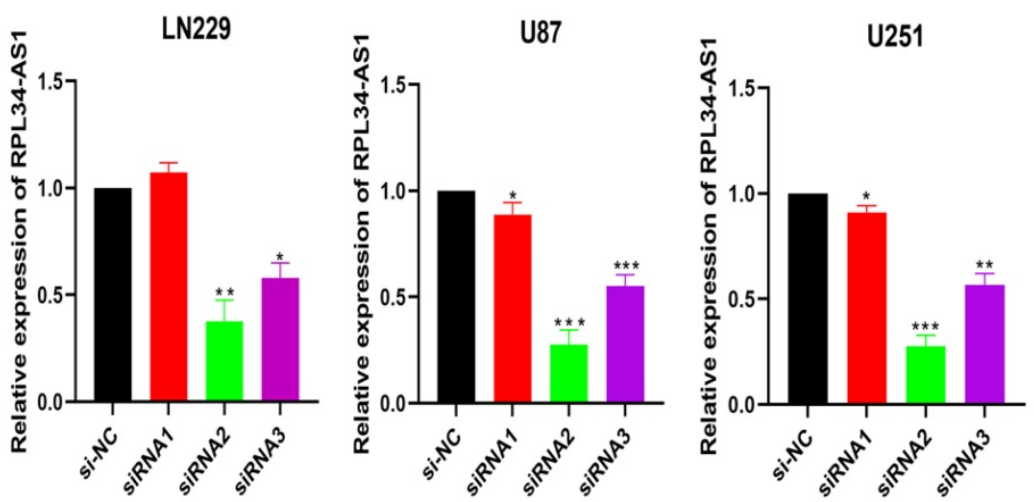

B

LN229

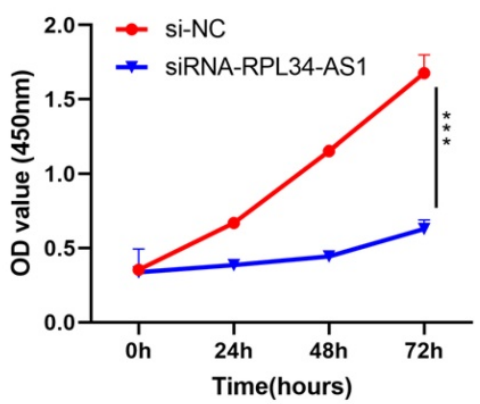

U251



U87

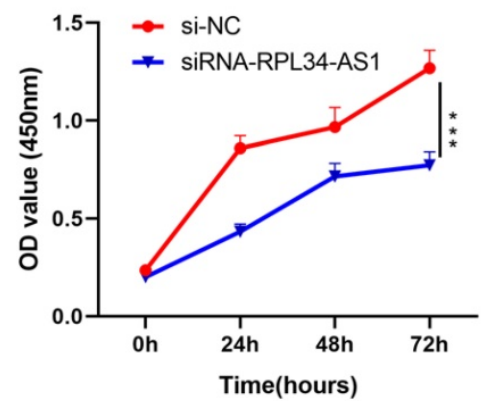


C

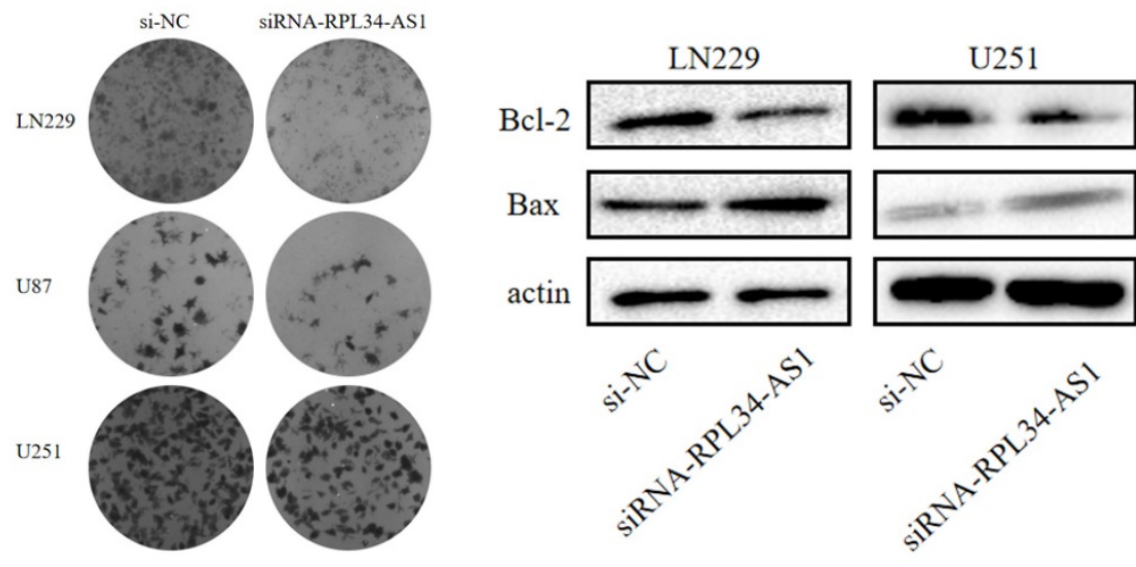

D
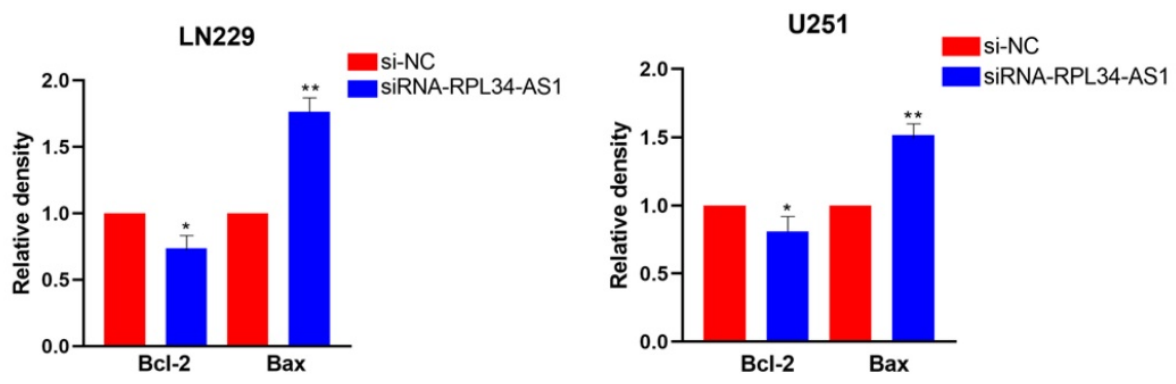

$\mathbf{E}$


Figure 2. Knockdown of RPL34-AS1 inhibits proliferation in glioma cell lines. (A) Decreased RPL34-AS1 level in LN229, U87 and U251 cell lines transfected with RPL34-ASI siRNAs compared with siRNA-NC. (B) Knockdown of RPL34-ASI impaired the proliferation of glioma cells compared with group of si-NC. (C) siRNA-RPL34-ASI inhibit colony formation of the glioma cell lines. (D) Western blot analysis revealed that siRNA-RPL34-AS1 increased Bax expression and decreased Bcl-2 expression. (E) Flow cytometric analysis also showed that silencing of RPL34-AS1 induced apoptosis in glioma cell lines $* \mathrm{P}<0.05 ; * * \mathrm{P}<0.01 ; * * * \mathrm{P}<0.001$.

\section{RPL34-AS1 promotes endothelial cell angiogenesis by regulating VEGFA}

Glioma cells can produce a large number of proangiogenic factors to stimulate angiogenesis, which helps to supply oxygen and nutrients to highly proliferative tumor cells [21]. Several clinical trials of antiangiogenic drugs in patients with newly diagnosed or recurrent gliomas have supported this view [22]. We conducted a biological analysis, which confirmed a positive correlation between RPL34-AS1 and VEGFA (Figure 3A). Therefore, we examined whether RPL34-AS1 has an effect on angiogenesis in glioma using a tube formation assay. The effect of
RPL34-AS1 knockdown on HUVECs was assessed by relative tube length. The conditioned media from si-RPL34-AS1 glioma cells exhibited a strong negative effect on HUVEC tube formation compared with that from si-NC cells (Figure 3B). We then measured the expression of VEGFA at the mRNA (Figure 3C) and protein levels (Figure 3D) using qRT-PCR and western blot, respectively.

\section{Knockdown of RPL34-ASI suppresses VEGFA production via regulation of ERK/AKT signaling}

Activation of ERK/AKT signaling in glioma cells plays an important role in indirectly activating 
VEGFA, which plays a crucial role in tumor

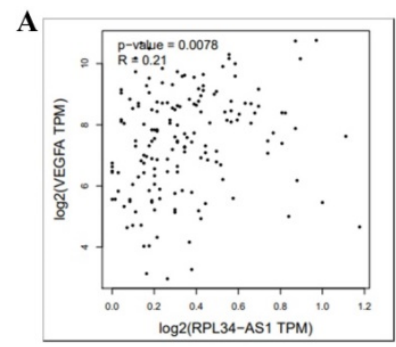

B
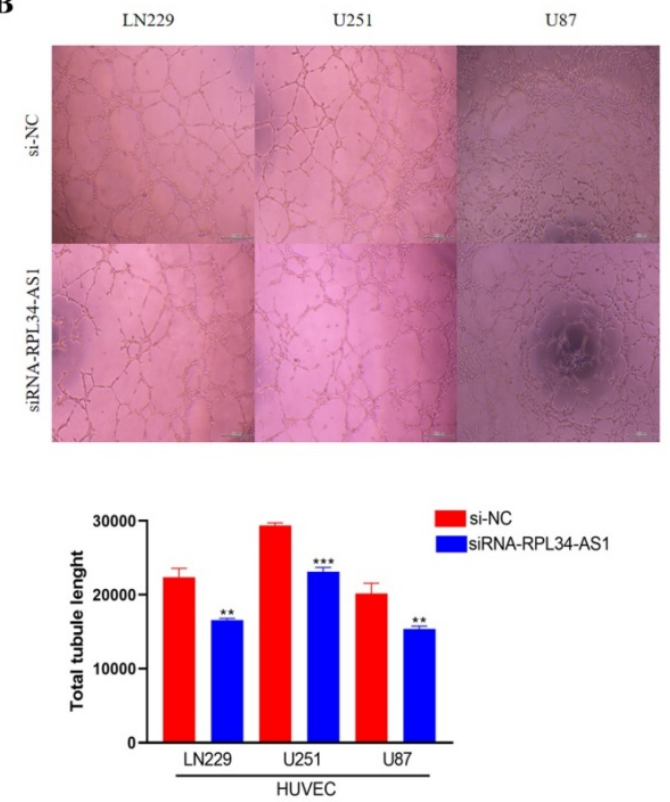

C


D


Figure 3. RPL34-ASI promotes endothelial cell angiogenesis by regulating VEGFA. (A) Biological analysis confirmed the positive correlation between RPL34-AS1 and VEGFA. (B) A tube formation assay was used to analyse the impact of RPL34-AS1 on HUVECs. (C, D) qRT-PCR and Western blotting assay were performed to detect the expression of VEGFA. *P < $0.05 ; * * \mathrm{P}<0.01 ; * * * \mathrm{P}<0.001$. angiogenesis $[23,24]$ through the regulation of various endothelial functions $[25,26,27]$. To confirm whether the contribution of RPL34-AS1 to glioma development was mediated through the ERK/AKT signaling pathway, we assessed the expression of ERK, AKT, p-ERK, and p-AKT by western blot (Figure 4A, 4B). The expressions of p-ERK and p-AKT were significantly decreased in cells transfected with si-RPL34-AS1 compared to cells transfected with si-NC, whereas there were no obvious changes observed in the expressions of total ERK and AKT.

\section{Discussion}

In this study, we report for the first time that the lncRNA RPL34-AS1 is highly expressed in gliomas, especially in HGGs. The expression of RPL34-AS1 was associated with poor prognosis and was related to glioma cell proliferation in vitro. We also proved that RPL34-AS1 mediates angiogenesis in glioma by regulating VEGFA. This study indicates that RPL34-AS1 may be a potential target for the treatment of glioma.

Glioma is the most common primary intracranial tumor and is derived from the carcinogenesis of glial cells in the neuroectoderm. Glioma accounts for $80 \%$ of primary intracranial malignant tumors [2]. It is characterized by excessive proliferation, recurrence, and high mortality. Surgery is currently the most important treatment for glioma, and with the rapid development of brain functional imaging, neuronavigation, electrophysiology, and other technologies, the maximum range of safe resection of tumors has become attainable. However, postoperative survival is not promising due to the biological characteristics of glioma. Because of the invasive nature of glioma, especially GBM, the median survival period is only 14.6 months, and the survival rate at 5 years after diagnosis is only $5.8 \%$ [3]. Therefore, it is necessary to study the pathogenesis of glioma at the molecular level to improve treatment options.

An increasing number of studies have reported that lncRNAs demonstrate remarkable functions in different tumors in terms of gene expression, mRNA regulation, protein modulation, and epigenetic regulation. Moreover, aberrant expression of lncRNAs in various human tumors has been demonstrated in recent years. There are significantly different lncRNA expression profiles in glioma tissue and normal brain tissue, and these differentially expressed 
RNAs are involved in glioma progression, complex conditions, and poor prognosis [21,28,29]. Here, we demonstrated that RPL34-AS1 expression was increased in glioma cell lines and glioma tissues, and RPL34-AS1 was significantly associated with WHO grade. Moreover, lncRNA RPL34-AS1 had a significant influence on cell proliferation and apoptosis, and silencing RPL34-AS1 reduced the proliferation of glioma cells in vitro. However, the mechanism by which it acts as an oncogene in glioma was still unknown.

Continuous angiogenesis has become the focus of our research as one of the biological hallmarks of tumors (Figure 5). It is well known that VEGFA is one of the most important factors in glioma angiogenesis. VEGFA has the capacity to induce physiological and pathological angiogenesis [30] to meet the nutrient
A
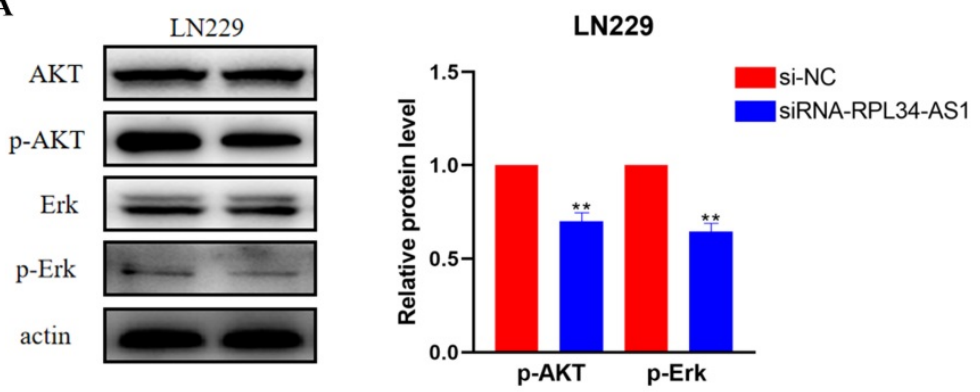

B



U251

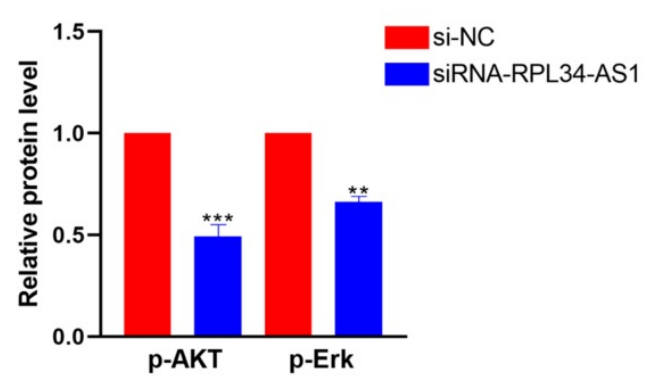

Figure 4. RPL34-AS1 exerts tumour promoter function through the Erk/AKT signaling. (A, B) Western blotting assay were performed to detect the expression on relative protein level of Erk/AKT signaling. *P $<0.05 ; * * \mathrm{P}<0.01 ; * * * \mathrm{P}<0.001$.

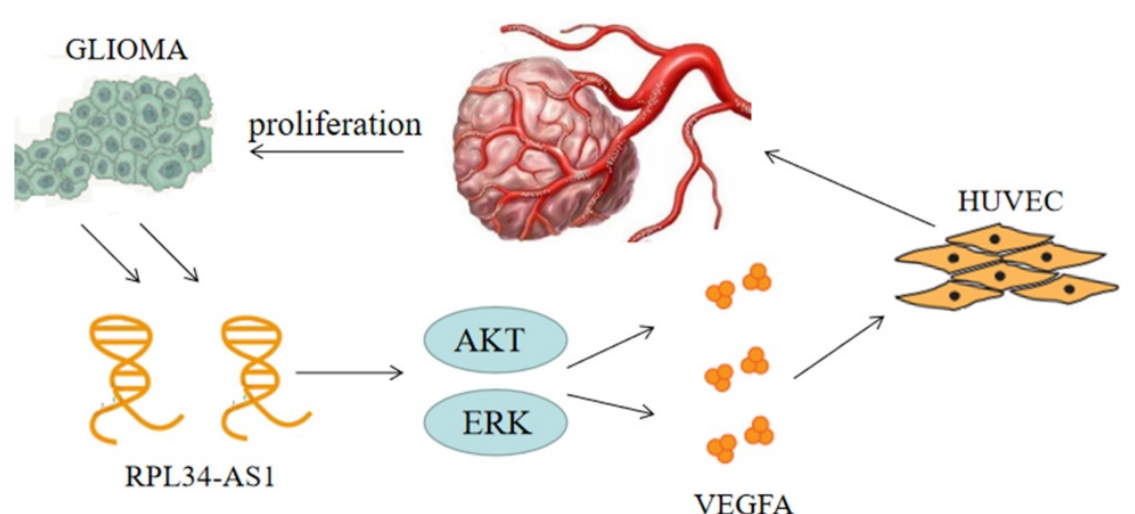

Figure 5. The schematic representation of the mechanism on IncRNA RPL34-AS1 to ErK/AKT/VEGFA axis in glioma proliferation and angiogenesis. needs of proliferating tumors. Glioma cells regulate angiogenesis through the angiogenic cytokine VEGFA, which signals to vascular endothelial cells in the tumor microenvironment [21]. This is a pathological characteristic of HGG. However, whether VEGFA is regulated by the lncRNA RPL34-AS1 remains largely unknown. Our biological analysis showed a positive correlation between RPL34-AS1 and VEGFA, suggesting that RPL34-AS1 may play a role in regulating VEGFA. Knockdown of RPL34-AS1 significantly decreased expression of VEGFA at the mRNA and protein levels, as well as tube formation of endothelial cells. Therefore, we conclude that RPL34-AS1 plays an important pathological role in glioma by affecting tumor angiogenesis.

Numerous signaling pathways have been implicated in VEGFA-induced endothelial cell proliferation, typically AKT and ERK, which activate glioma cells and indirectly activate VEGFA through their action on HIF-1a [31,32]. Previous studies have found that when the ERK/AKT pathway is active, it can effectively promote the proliferation and invasion of tumor cells, and this process is closely related to VEGFA [33]. ERK signaling is the main pathway involved in signal transduction in vascular endothelial cells [34]. In addition, the AKT signaling pathway, which can promote the transcription of VEGFA, is widely active in brain tissue. This transcription stimulates the production of VEGFA to promote angiogenesis [35]. Furthermore, we have previously shown that silencing RPL34-AS1 led to a significant change in EGFR expression. As the upstream gene of the ERK/AKT pathway, EGFR is closely related to the proliferation, invasion, and migration of tumor cells [36]. The specific mechanism will be the aim of our future research.

In conclusion, our study showed that RPL34-AS1 expression is upregulated in glioma tissues and cell lines. Knockdown of RPL34-AS1 suppresses proliferation and induces apoptosis in glioma cell lines. RPL34-AS1 exerts oncogenic activity 
partially by affecting angiogenesis in glioma by regulating the VEGFA and ERK/Akt signaling pathways. Therefore, RPL34-AS1 may be a potential novel target for glioma therapy.

\section{Abbreviations}

LGG: low-grade glioma; HGG: high-grade glioma; VEGFA: vascular endothelial growth factor A; IncRNA: Long non-coding RNA; PI3K: phosphoinositide 3-kinase; AKT: Serine/threonine-protein kinase; ERK: extracellular regulated protein kinases; RPL34-AS1: ribosomal protein L34 antisense RNA 1; RPL34: ribosomal protein L34; NHAs: normal human astrocytes; GBM: glioblastoma multiforme; HUVECs: human umbilical vein endothelial cells; HIF-1a: hypoxia inducible factor-1a; EGFR: epidermal growth factor receptor.

\section{Acknowledgements}

We would like to thank Editage (www. editage.cn) for English language editing.

\section{Funding}

This work was supported by the National Natural Science Foundation of China (grant number 81972363).

\section{Competing Interests}

The authors have declared that no competing interest exists.

\section{References}

1. Weller M, Wick W, Aldape K, et al. Glioma. Nat Rev Dis Primers. 2015; 15017

2. Molinaro AM, Taylor JW, Wiencke JK, et al. Genetic and molecular epidemiology of adult diffuse glioma. Nat Rev Neurol. 2019; 15(7).

3. Tan AC, Ashley DM, Giselle Y. López, et al. Management of glioblastoma: State of the art and future directions. Ca Cancer J Clin. 2020; 70(15).

4. Aldape K, Brindle KM, Chesler L, et al. Challenges to curing primary brain tumours. Nat Rev Clin Oncol. 2019; 41571-019-0177-5.

5. Apte RS, Chen DS, Ferrara N. VEGF in Signaling and Disease: Beyond Discovery and Development. Cell. 2019; 176(6): 1248-1264

6. Hai-Li Lang, et al. Glioma cells enhance angiogenesis and inhibit endothelial cell apoptosis through the release of exosomes that contain long non-coding RNA CCAT2. Oncol Rep. 2017; 38(2): 785-798.

7. Duda DG, Loeffler JS, Sorensen AG, et al. Angiogenesis in brain tumours. Nat Rev Neurosc. 2007; 8(8): 610-622.

8. Baumgarten $\mathrm{P}$, Blank AE, Franz $\mathrm{K}$, et al. Differential expression of vascular endothelial growth factor $\mathrm{A}$, its receptors VEGFR-1, -2 , and -3 and co-receptors neuropilin-1 and -2 does not predict bevacizumab response in human astrocytomas. Neuro Oncol. 2016; 18(2): 173-183.

9. Ye J, Zhu J, Chen $\mathrm{H}$, et al. A novel IncRNA-LINC01116 regulates tumorigenesis of glioma by targeting VEGFA. Int J Cancer. 2020; 146(1).

10. Jiang $Y$, Zhou J, Zhao J, et al. The U2AF2 / circRNA ARF1/miR-342-3p/ISL2 feedback loop regulates angiogenesis in glioma stem cells. J Exp Clin Cancer Res. 2020; 39(1): 182.

11. Peng WX, Koirala P, Mo YY. LncRNA-mediated regulation of cell signaling in cancer. Oncogene. 2017; 17: 0950-9232.

12. Bhan A, Soleimani M, Mandal SS. Long Noncoding RNA and Cancer: A New Paradigm. Cancer Res. 2017; 16: 2634

13. Wang $\mathrm{Y}$, Wang $\mathrm{Y}, \mathrm{Li}$ J, et al. CRNDE, a long-noncoding RNA, promotes glioma cell growth and invasion through mTOR signaling. Cancer Lett. 2015; 367(2): 122-128.

14. Zhou Q Liu ZZ, Wu $\mathrm{H}$, et al. LncRNA H19 Promotes Cell Proliferation, Migration, and Angiogenesis of Glioma by Regulating Wnt5a/ $\boldsymbol{\beta}$-Catenin Pathway via Targeting miR-342. Cell Mol Neurobiol. 2020; 1-13.
15. Yan $Y, X u Z$, Chen $X$, et al. Novel Function of IncRNA ADAMTS9-AS2 in Promoting Temozolomide Resistance in Glioblastoma via Upregulating the FUS/MDM2 Ubiquitination Axis. Front Cell Dev Biol. 2019; 7: 217.

16. Jiang $\mathrm{X}, \mathrm{Yan} \mathrm{Y}, \mathrm{Hu} \mathrm{M}$, et al. Increased level of H19 long noncoding RNA promotes invasion, angiogenesis, and stemness of glioblastoma cells. J Neurosurg. 2016; 124: 129-136.

17. Ke J, Yao YL, Zheng J, et al. Knockdown of long non-coding RNA HOTAIR inhibits malignant biological behaviors of human glioma cells via modulation of miR-326. Oncotarget. 2015; 6: 26.

18. Gong Z, Li J, Cang P, et al. RPL34-AS1 functions as tumor suppressive lncRNA in esophageal cancer. Biomed Pharmacother. 2019; 120: 0753-3322.

19. Ji L, Fan X, Zhou F, et al. IncRNA RPL34-AS1 inhibits cell proliferation and invasion while promoting apoptosis by competitively binding miR-3663-3p/ RGS4 in papillary thyroid cancer. J Cell Physiol. 2020; 235(4).

20. Jing, Zhao, Yongchao, et al. Long non-coding RNA Linc00152 is involved in cell cycle arrest, apoptosis, epithelial to mesenchymal transition, cell migration and invasion in gastric cancer. Cell cycle. 2015; 14(19): 3112-23.

21. Hai-Bo, Wu, Shuai, et al. Autophagy-induced KDR/VEGFR-2 activation promotes the formation of vasculogenic mimicry by glioma stem cells. Autophagy. 2017; 13( 9): 1528-1542.

22. Khasraw M, Ameratunga MS, Grant R, et al. Antiangiogenic therapy for highgrade glioma. Cochrane Database Syst Rev. 2014; 9(9): CD008218.

23. Wang C, Li Y, Chen H, et al. Inhibition of CYP4A by a novel flavonoid FLA-16 prolongs survival and normalizes tumor vasculature in glioma. Cancer Lett. 2017; 1/11: 0304-3835.

24. Aditi, Jain, James CK, et al. Biochanin A inhibits endothelial cell functions and proangiogenic pathways: implications in glioma therapy. Anticancer Drugs. 2015; $26: 3$.

25. Manning BD, Cantley LC. AKT/PKB Signaling: Navigating Downstream. Cell. 2007; 129(7): 1261-1274.

26. Koide $\mathrm{M}$, Ikeda $\mathrm{K}$, Akakabe $\mathrm{Y}$, et al. Apoptosis regulator through modulating IAP expression (ARIA) controls the PI3K/Akt pathway in endothelial and endothelial progenitor cells. Proc Natl Acad Sci USA. 2011; 108(23): 9472-9477.

27. Ichiro Shiojima, Kenneth Walsh. Role of Akt signaling in vascular homeostasis and angiogenesis. Circulation Research. 2002; 6(28).

28. Wu J, Guo X, Xu D, et al. LINC00662 sponges miR-107 accelerating the invasiveness and proliferation of glioma cells. J Cancer. 2020; 11(19): 5700-5712.

29. Ke Z, Chi Z, Hui Y, et al. Knockdown of long non-coding RNA NEAT1 inhibits glioma cell migration and invasion via modulation of SOX2 targeted by miR-132. Mol Cancer. 2018; 17(1): 105.

30. Goel HL, Mercurio AM. VEGF targets the tumour cell. Nat Rev Cancer. 2013; 13(12): 871-882.

31. Semenza G. HIF-1 and tumor progression: pathophysiology and therapeutics. Trends Mol Med. 2016; 8(4): S62-S67.

32. Testini C, Smith RO, Jin Y, et al. Myc-dependent endothelial proliferation is controlled by phosphotyrosine 1212 in VEGF receptor-2. Embo Rep. 2020; 21(5): e50409.

33. Milosevic Z, Pesic M, Stankovic T, et al. Targeting RAS-MAPK-ERK and PI3K-AKT-mTOR signal transduction pathways to chemosensitize anaplastic thyroid carcinoma. Transl Res. 2014; 164(5): 411-423.

34. Shen SW, Duan CL, Chen XH, et al. Neurogenic effect of VEGF is related to increase of astrocytes transdifferentiation into new mature neurons in rat brains after stroke. Neuropharmacology. 2016; 108: 451-461.

35. Lin LI, Hao J, Jiang $X$, et al. Cardioprotective effects of ulinastatin against isoproterenol-induced chronic heart failure through the PI3KAkt, p38 MAPK and NF-kB pathways. Mol Med Rep. 2017; 17(1).

36. Lu Y, Feng J, Jiang H, et al. Gallic acid suppresses cell viability, proliferation, invasion and angiogenesis in human glioma cells. Eur J Pharmacol. 2010; 641(2): 102-107. 